\title{
Orbital precursor B-lymphoblastic lymphoma involving the extraocular muscles in a 56-year-old male and a review of the literature
}

\author{
RASMUS EJSTRUP ${ }^{1}$, LAUGE HJORTH MIKKELSEN ${ }^{1,2}$, METTE KLARSKOV ANDERSEN ${ }^{3}$, \\ ERIK CLASEN-LINDE ${ }^{2}$, LISE METTE RAHBEK GJERDRUM ${ }^{4}$, SETAREH SAFAVI $^{3}$ and STEFFEN HEEGAARD ${ }^{1,2}$ \\ Departments of ${ }^{1}$ Ophthalmology, ${ }^{2}$ Pathology and ${ }^{3}$ Clinical Genetics, Rigshospitalet, Copenhagen University Hospital, \\ 2100 Copenhagen; ${ }^{4}$ Department of Pathology, Zealand University Hospital, 4000 Roskilde, Denmark
}

Received January 16, 2018; Accepted September 26, 2018

DOI: $10.3892 / \mathrm{ol} .2018 .9725$

\begin{abstract}
The aim of the present study was to describe a rare case of orbital precursor B-lymphoblastic lymphoma (B-LBL) in an adult. A 56-year-old male in complete remission of a gastric precursor B-LBL was referred to our orbital clinic due to rapid development of left-sided painless periorbital swelling, diplopia, and proptosis. Complete ophthalmoplegia was observed. Notably, magnetic resonance imaging showed swelling of the medial and inferior rectus muscles in the left orbit and biopsies were performed. Following histological diagnosis of precursor B-LBL, the patient was treated with radiotherapy (2Gy x 20) and chemotherapy according to the NOPHO ALL 2008 protocol. The disease progressed and the patient succumbed after 5 months. Histomorphologically, a lymphoblastic infiltrate was observed within the skeletal muscle tissue. The tumor cells were small and immature, and stained strongly for cluster of differentiating (CD)10, CD79a, paired box 5 and B cell lymphoma-2. The Ki- 67 proliferative index was $90 \%$. Multiplex ligation-dependent probe amplification and array comparative genomic hybridization detected whole chromosomal gain of $\mathrm{X}$ and 12 , and both hemizygous and homozygous deletion on $9 \mathrm{p}$ comprising cyclin dependent kinase inhibitor 2A/B. Furthermore, array comparative genomic hybridization detected copy number imbalances consisting of focal or smaller deletions on chromosomes 1 , $9,10,11$ and 20. The final diagnosis was precursor B-LBL relapse in the extraocular muscles. Orbital precursor B-LBL is extremely rare in adults, and the diagnosis may be challenging to make. It is recommended to obtain material for cytogenetic and molecular analyses.
\end{abstract}

Correspondence to: Professor Steffen Heegaard, Department of Pathology, Rigshospitalet, Copenhagen University Hospital, 11-1 Frederik V's Vej, 2100 Copenhagen, Denmark

E-mail: sthe@sund.ku.dk

Key words: precursor, lymphoblastic lymphoma, lymphoma, orbit, extraocular muscles

\section{Introduction}

Precursor B-lymphoblastic lymphoma (B-LBL) is a rare and highly aggressive malignant neoplasm of lymphoblasts committed to the B-cell lineage, primarily presenting as extranodal tumors and with low propensity for leukemic involvement in children and young adults (1). B-LBL is an uncommon subtype of ocular adnexal lymphoma (OAL), with only nine published orbital cases (Table I). Of the previous cases, one occurred in an infant (2), five in children (3-7), and two in young adults (6). OAL is the most frequent orbital malignancy in adults (6); however, extraocular muscle involvement is uncommon. In 2011, Watkins et al (8) published a major review of lymphoid tumors in extraocular muscles, identifying 57 cases comprising $0.17 \%$ of all OAL. Since then, eight additional cases have been published (9-12). So far, none of the reported cases presented as B-LBL.

We present the first case of B-LBL with extraocular muscle involvement. The patient is older than the normal age range for this disease and showed intermittent orbital symptoms initially undetectable by magnetic resonance imaging (MRI). The aim of this study was to present the clinical, histopathological, and genetic characteristics of this rare condition.

\section{Materials and methods}

Clinical course. A 56-year-old Caucasian male was in complete remission of a histopathologically confirmed precursor B-LBL in the abdomen treated with chemotherapy (Hyper-CVAD) according to the MD Anderson recommendations (13). Remission was confirmed by whole body positron emission tomography-computerized tomography (PET-CT) and CT of the abdomen. At this time, flow cytometry of a bone marrow biopsy showed many suspected malignant CD10 and CD19 positive cells from the B-cell lineage, representing immature B-cells.

Three months later, the patient experienced a sudden onset of left-sided droopy eyelid and diplopia without pain for a few days. These symptoms disappeared spontaneously and the neurological examination was unremarkable. MRI of the orbit 
did not detect a mass lesion and ophthalmological examination was normal.

Another 2 months later, the symptoms reoccurred with marked periorbital swelling, blepharoptosis, diplopia, and slight proptosis of the left eye without pain. Both MRI of the orbit and PET were again normal and the patient was referred to our tertiary orbital clinic. On examination, left axial proptosis (Hertel 22/26 mm) and partial left-side third nerve palsy was noted. Visual acuity (VA) was $6 / 6$ in both eyes. Color vision, pupil response, and visual field investigation were all normal. However, during the following 5 days, rapidly developing pain, decreased visual acuity of the left eye $(6 / 12)$, chemosis, increasing proptosis $(22 / 27 \mathrm{~mm})$, and total ophthalmoplegia (frozen socket) ensued. Mydriasis developed, but no afferent pupillary (RAPD) defect was present. MRI showed thickening of the medial and inferior rectus muscles with very subtle 'dirty fat', suggesting an infiltrative lesion (Fig. 1A). Orbital biopsies were obtained through an anterior orbitotomy. The patient had no light perception the day after surgery. A blood test performed at the time of orbital involvement was normal with no blasts. At diagnosis, repeated bone marrow biopsies showed no systemic involvement. Chemotherapy according to the high-risk NOPHO ALL 2008 protocol (14) and orbital radiation (2Gy x 20) was initiated and myeloablative allogeneic bone marrow transplantation was planned.

One month postoperatively, the orbital swelling diminished and some abduction was noted. However, the patient's condition quickly deteriorated to a degree where treatment was not tolerated. A bone marrow biopsy at this time revealed systemic involvement with numerous blasts present. The patient ceased before transplantation was performed.

The patient has given his informed consent to publication. The present study adheres to the declaration of Helsinki, and was approved by the local ethics committee (H-B-2009-054).

Histopathology and immunohistochemistry. A biopsy from the inferior rectus muscle of the left orbit was obtained for histological examination. The specimen was fixed in $10 \%$ formalin and embedded in paraffin immediately after surgical excision. Sections $(2 \mu \mathrm{m})$ were prepared and stained with hematoxylin and eosin according to standard protocols. Furthermore, sections were stained with periodic acid Schiff (PAS), Gram, Gomori methenamin silver stain, and Giemsa also in line with standard protocols.

Immunohistochemical stainings were performed on a Ventana BenchMark ULTRA Platform (Ventana Medical Systems, Tucson, AZ, USA) as previously described (15). The following antibodies were used: Cluster of differentiation (CD) 3 (clone 2GV6, code 790-4341, rabbit anti-human, Ready-to-use (RTU); Roche, Hvidovre, Denmark), CD10 (clone SP 67, code 790-4506, rabbit anti-human, RTU; Roche), CD20 (clone L26, code 760-2531, mouse anti-human, RTU; Roche), CD34 (clone QBEnd/10, code 790-2927, mouse anti-human, RTU; Roche), CD79a (clone SP18, code 790-4432, rabbit anti-human, RTU; Roche), BCL-2 (clone 124, code 790-4464, mouse anti-human, RTU; Roche), BCL6 (clone GI1E/A8, code 760-4241, mouse anti-human, RTU; Roche), MPO (polyclonal, code A0398, rabbit anti-human, 1:2,000; Dako; Agilent Technologies, Inc., Santa Clara, CA, USA), PAX-5 (clone
SP34, code 790-4420, rabbit anti-human, RTU; Roche), TdT (clone SEN28, code NCL-TdT-339, mouse anti-human, 1:100; Novocastra, Brønshøj, Denmark), Vimentin (clone V9, code 790-2917, mouse anti-human, RTU; Roche), and KI-67 (clone MIB-1, code M724001, mouse anti-human, 1:100; Dako; Agilent Technologies, Inc.). Positive and negative controls were performed according to the manufacturers' instructions.

Genetics. After dissecting the tumor sample, genomic DNA and RNA were extracted using QIAamp DNA Mini kit (Qiagen GmbH, Hilden, Germany).

Multiplex ligation-dependent probe amplification. Multiplex ligation-dependent probe amplification (MLPA) was performed on DNA using the SALSA MLPA kit P335-A1 according to manufacturer's instructions (MRC Holland, Amsterdam, the Netherlands). The analysis is suited for the detection of copy number variations in the following genes: $I K Z F 1, C D K N 2 A / B$, $P A X 5, E B F 1, E T V 6, R B 1, B T G 1, R B 1$, and the PAR1 region: CRLF2, CSF 2A, IL3A.

Array $\mathrm{CGH}$. Genome-wide array $\mathrm{CGH}$ analysis (array $\mathrm{CGH}$ ) was performed on DNA using the Agilent SurePrint G3 Human CGH Microarray kit 2x400K (Agilent Technologies, Inc.). Labeling and hybridization were performed according to the protocol provided by Agilent Technologies, Inc., (protocol v6.0, November 2008) as previously described (16).

Multiplex RT-PCR. Multiplex RT-PCR was performed on cDNA synthesized from tumor RNA according to the HemaVision-28N protocol (HemaVision-28N, Cat. No. HV01-28N, DNA Diagnostic, Risskov, Denmark). The analysis screens for 28 chromosomal translocations, including the most common ALL related translocations.

Fluorescent in situ hybridization (FISH). Fluorescent in situ hybridization (FISH) was performed using the probes for BCR-ABL (cat. no: 05J90-001; Abbott Molecular, Emdrup, Denmark), ETV-6 (cat. no: Z-2176; ZytoVision GmbH, Bremerhaven, Germany), and MLL (KMT2A) (cat. no: Z-2111; ZytoVision) according to the manufacturers' protocol using the HyBrite platform (Abbott Molecular). After hybridization, the nuclei were counterstained with DAPI II (ZytoVision). One hundred nuclei were counted and the cut-off was set to $10 \%$.

Flow cytometry. The BM sample was processed and stained for flow cytometric immunophenotyping with standard procedures according to the NOPHO acute lymphoblastic leukemia guidelines (17). Data acquisition was performed on a BD FACSCanto II (BD Biosciences, San José, CA, USA) according to manufacturer's instructions. An antibody combination of CD3 (clone: G4.18, cat. no: 554829, RTU; BD Biosciences), CD4 (clone: SK3, cat. no: 651949, RTU; BD Biosciences), CD5 (clone: L17F12, cat. no: 341109, RTU; BD Biosciences), CD8 (clone: SK1, cat. no: 345772, RTU; BD Biosciences), CD10 (clone: ALB1, cat. no: IM1915u, RTU; Beckman Coultier, Copenhagen, Denmark), CD19 (clone: SJ25C1, cat. no: 341113 , RTU; BD Biosciences), CD20 (clone: L27, cat. no: 655872, RTU; BD Biosciences), CD23 (clone: MHM6, cat. no: F7062, RTU; Dako; Agilent Technologies, Inc.), CD34 (clone: 581, 
Table I. Cases of biopsy-proven orbital precursor-lymphoblastic lymphoma/acute lymphoblastic leukemia reported in the literature.

\begin{tabular}{|c|c|c|c|c|c|c|c|}
\hline Author (year) & $\begin{array}{c}\text { Age } \\
\text { (years) }\end{array}$ & $\begin{array}{l}\text { Primary } \\
\text { lesion }\end{array}$ & $\begin{array}{l}\text { Sex } \\
m / f\end{array}$ & Location & Treatment & Prognosis & (Refs.) \\
\hline Thakker et al (2006) & $<1$ & Yes & $\mathrm{F}$ & Right orbit & NOPHO & $\begin{array}{l}\text { Alive } 1 \text { year } \\
\text { without relapse }\end{array}$ & (2) \\
\hline Chou et al (2005) & 6 & No & M & Left orbit & $\begin{array}{l}\text { RT and } \\
\text { chemotherapy }\end{array}$ & $\begin{array}{l}\text { Alive } 4 \text { years } \\
\text { without relapse }\end{array}$ & (3) \\
\hline Alford et al (1999) & 12 & Yes & M & Left orbit & $\begin{array}{l}\text { RT and } \\
\text { chemotherapy } \\
\text { and ASCT }\end{array}$ & $\begin{array}{l}\text { Multiple relapses, } \\
\text { alive } 4 \text { years } \\
\text { after last relapse }\end{array}$ & (4) \\
\hline Faridpooya et al (2009) & 6 & Yes & M & Left orbit & Chemotherapy & $\begin{array}{l}\text { Alive } 19 \text { years } \\
\text { without relapse }\end{array}$ & (5) \\
\hline Ferry et al (2007) & 7 & Yes & $\mathrm{F}$ & Left orbit & N/A & N/A & (6) \\
\hline Ferry et al (2007) & 38 & Yes & $\mathrm{F}$ & Left orbit & N/A & N/A & (6) \\
\hline Ferry et al (2007) & 45 & Yes & $\mathrm{F}$ & Right orbit & N/A & N/A & (6) \\
\hline $\begin{array}{l}\text { Ramamoorthy } \\
\text { et al (2016) }\end{array}$ & 4 & No & $\mathrm{F}$ & Left orbit & Chemotherapy & $\begin{array}{l}\text { Alive } 6 \text { months } \\
\text { without relapse }\end{array}$ & (7) \\
\hline Present case & 56 & No & M & $\begin{array}{l}\text { Left orbit, } \\
\text { extraocular } \\
\text { muscles }\end{array}$ & $\begin{array}{l}\text { RT and } \\
\text { NOPHO }\end{array}$ & $\begin{array}{l}\text { Died after } \\
3 \text { months }\end{array}$ & - \\
\hline
\end{tabular}

M, male; F, female; RT, radiotherapy; ASCT, allogenic stem cell transplantation.

cat. no: A89309, RTU; Beckman Coultier), CD38 (clone: HB7, cat. no: 345807, RTU; BD Biosciences), CD45 (clone: 2D1, cat. no: 655873, RTU; BD Biosciences), CD56 (clone: NCAM 16.2, cat. no: 345812, RTU; BD Biosciences), CD79b (clone: SN8, cat. no: 335834, RTU; BD Biosciences), CD200 (clone: OX104, cat. no: 17-9200-41, RTU, eBioscience, Thermo Fischer Scientific, Roskilde, Denmark), kappa light chain (Polyclonal, cat. no: R0436, RTU; Dako; Agilent Technologies, Inc.), and lambda light chain (Polyclonal, cat. no: F0435, RTU; Dako; Agilent Technologies, Inc.), were used. Data was analyzed using FlowJo 9.3.1 software (FlowJo LLC, Ashland, OR, USA) according to the NOPHO protocol (17).

\section{Results}

Histopathology and immunohistochemistry. An infiltrate of immature lymphoblastic cells was seen within the orbital skeletal muscle tissue (Fig. 1B). The tumor cells were small and presented with fine blastic nuclear chromatin along with small peripheral nucleoli (Fig. 1C). The tumor cells stained positively for PAX-5 (Fig. 1D) CD10, CD79a, and BCL-2. Staining for CD3, CD20, CD34, TdT, BCL6, and MPO was negative. The Ki-67 proliferation index was very high, approximately $90 \%$ (Fig. 1E). These findings resembled the primary abdominal biopsy and were consistent with a TdT-negative precursor B-LBL relapse.

Biopsies obtained from the orbital soft tissue contained no tumor cells, indicating that the tumor was confined to the orbital muscles.
Flow cytometry. Flow cytometry of a bone marrow biopsy performed at the time of first presentation identified numerous monoclonal immature blasts from the B-cell lineage (Fig. 2).

Genetics. FISH analyses were negative for rearrangement of ETV6, KMT2A (formerly $M L L$ ), and $B C R / A B L$.

MLPA and array CGH analyses detected whole chromosomal gain of $\mathrm{X}$ and 12 and both hemizygous and homozygous deletion on 9p comprising $C D K N 2 A / B$ (deletions ranging from 21,323,778-30,864,756 bp; Fig. 3). Array CGH showed that the pattern of deletions was complex, with homozygous deletions flanked by hemizygously deleted regions (Fig. 4).

Furthermore, array CGH detected copy number imbalances consisting of focal or smaller deletions on chromosomes 1, 9, 10, 11, and 20 (Table II).

No leukemia-associated translocations were identified when screening for 28 chromosomal translocations by multiplex RT-PCR.

\section{Discussion}

The present study presents the first case of B-LBL of the orbit involving the extraocular muscles in a 56-year-old male.

Precursor B-LBL has a male predilection, is primarily a disease of children and young adults, and the clinical course is usually characterized by rapid growth of a mass involving lymph nodes, skin, bone, or other extranodal sites. The prognosis is favorable, with a survival rate of approximately $90 \%$ in 
A

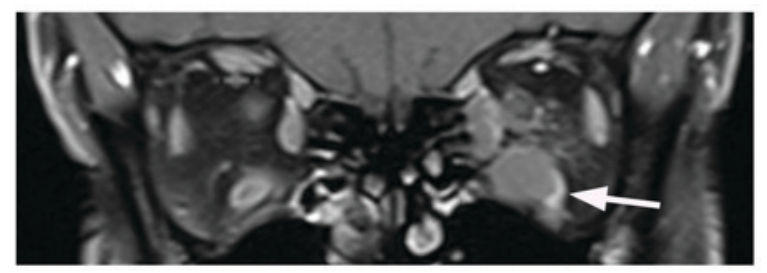

B

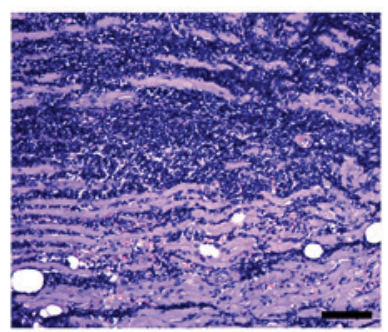

D

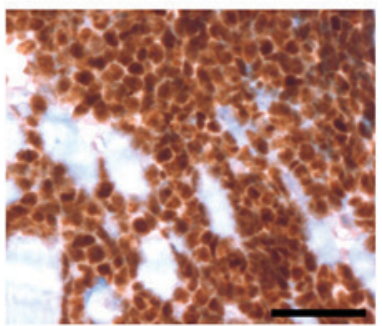

$\mathbf{E}$
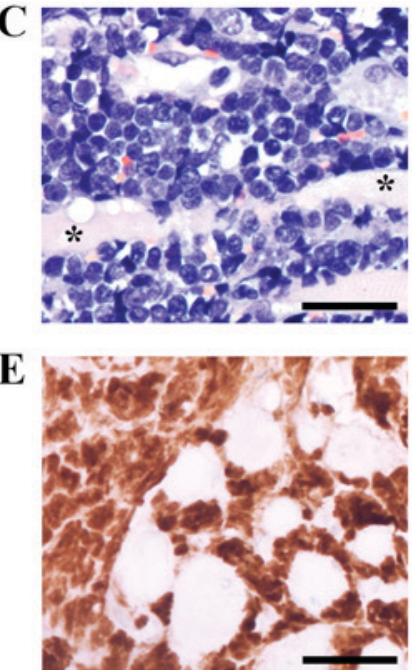

Figure 1. Magnetic resonance imaging and histopathological analysis of orbital B-LBL. (A) Magnetic resonance imaging showed swelling of the left inferior and medial rectus muscles (arrow) and a biopsy was performed. (B) A neoplastic infiltrate was found within the skeletal muscle tissue (hematoxylin and eosin; bar $=100 \mu \mathrm{m}$ ). (C) The small tumor cells had a blastic morphology and surrounded the myocytes (asterisk). The cytoplasm was scant and the nucleoli had a fine chromatin (hematoxylin and eosin; bar=40 $\mu \mathrm{m}$ ). (D) The tumor cells were positive when stained for the pan-B-cell marker PAX-5 (bar=50 $\mu \mathrm{m})$. (E) The proliferative index measured with Ki-67 was approximately $90 \%$ (bar=70 $\mu \mathrm{m}$ ). B-LBL, B-lymphoblastic lymphoma.

children, but outcome worsens with age (1), as the present case illustrates. In addition, patients with relapsed disease have a poor outcome (18). This is the oldest patient described with orbital B-LBL and a corresponding aggressive clinical course. Despite appropriate treatment with orbital radiotherapy and multi-agent chemotherapy according to high-risk NOPHO regimen (12), the disease was refractory and the patient died. Eight previously reported cases of biopsy-proven orbital B-LBL are summarized in Table I. B-LBL and B-acute lymphoblastic leukemia (ALL) are considered the same disease spectrum, distinguished by the degree of bone marrow involvement (1). Whereas extraocular muscle involvement of an orbital T-lymphoblastic lymphoma has recently been described (12), this is the first reported case with primarily extraocular muscle involvement of B-LBL. Thus, representing a rare manifestation of orbital lymphoma, this case highlights the importance of ensuring an accurate diagnosis by means of an orbital biopsy, including the extraocular muscles if indicated by imaging. Indeed, the extramuscular orbital biopsy was devoid of lymphoblasts, which were only confirmed in the extraocular muscles.

The histopathological diagnosis of a highly aggressive orbital B-LBL may be challenging and may lead to delayed initiation of the correct therapy. The immature tumor cells may present with slightly different morphology and immunohistochemistry may not always be conclusive. A positive staining for TdT is usually suggestive for the diagnosis of
Table II. Imbalances detected by array comparative genomic hybridization.

\begin{tabular}{rcl}
\hline Chromosome & Position (bp) & \multicolumn{1}{c}{ Gain/loss } \\
\hline 1 & $188,838,117-190,329,277$ & Hemizygous loss \\
1 & $214,360,104-214,736,822$ & Hemizygous loss \\
1 & $232,437,508-236,208,925$ & Hemizygous loss \\
9 & $10,631,808-15,671,229$ & Hemizygous loss \\
9 & $21,323,778-21,885,253$ & Hemizygous loss \\
9 & $21,901,322-22,032,716$ & Homozygous loss \\
9 & $22,036,446-22,365,771$ & Hemizygous loss \\
9 & $22,382,290-22,583,476$ & Homozygous loss \\
9 & $22,592,720-26,554,414$ & Hemizygous loss \\
9 & $26,576,505-26,746,962$ & Homozygous loss \\
9 & $26,760,476-30,864,756$ & Hemizygous loss \\
9 & $75,777,676-79,018,498$ & Hemizygous loss \\
10 & $9,551,403-11,770,319$ & Hemizygous loss \\
10 & $37,205,162-37,826,531$ & Hemizygous loss \\
11 & $6,550,803-6,632,573$ & Hemizygous loss \\
11 & $6,639,238-6,665,699$ & Homozygous loss \\
11 & $6,669,807-6,724,677$ & Hemizygous loss \\
12 & pter-qter & Gain \\
20 & $57,595,945-58,000,098$ & Hemizygous loss \\
$X$ & pter-qter & Gain \\
\hline & &
\end{tabular}

ALL/LBL, but in the present case this staining was negative. Interestingly, a case of a TdT-negative orbital T-LBL with muscle involvement has previously been described (12).

Molecular techniques are needed to make a genetic diagnosis allowing for more precise characterization of the tumor in case of diagnostic uncertainty. The eye pathologist should preferably obtain unfixed material for this purpose. In the present case, array CGH, FISH, and MLPA were performed. The tumor had an unstable genome with whole chromosomal gain of $\mathrm{X}$ and 12, and focal or smaller deletions were found on chromosomes 1, 9, 10, 11, and 20. Furthermore, both hemizygous and homozygous deletion on $9 \mathrm{p}$, comprising $C D K N 2 A / B$ was detected. Several cytogenetic abnormalities have been identified in B-LBL, and these are highly valuable in the prognostication of paediatric disease (19). In contrast, adult cases have not been studied in detail, and good prognostic features such as hyperdiploidy with 51-65 chromosomes and the translocation $\mathrm{t}(12 ; 21)$ are infrequent. Whole chromosomal gain of the sex chromosomes has been shown to be common in adult pre-B ALL, and deletions of $C D K N 2 A / B$ are highly characteristic of LBL/ALL in all age groups in hemizygous and homozygous forms (20). Inactivation of the tumor suppressor genes, $C D K N 2 A / B$, is associated with a poor outcome in all B-LBL patients (21). This is in line with the poor outcome in our case. In adult B-LBL, the poor prognostic translocation $\mathrm{t}(9 ; 22)(\mathrm{q} 34 ; \mathrm{q} 11.2)$ $(B C R / A B L)$ is found in $25 \%$ of cases (19), however, this was not present in our case.

As the present case illustrates, patients with relapsed $\mathrm{B}-\mathrm{LBL}$ or presentation at a higher age have a poor prognosis. 

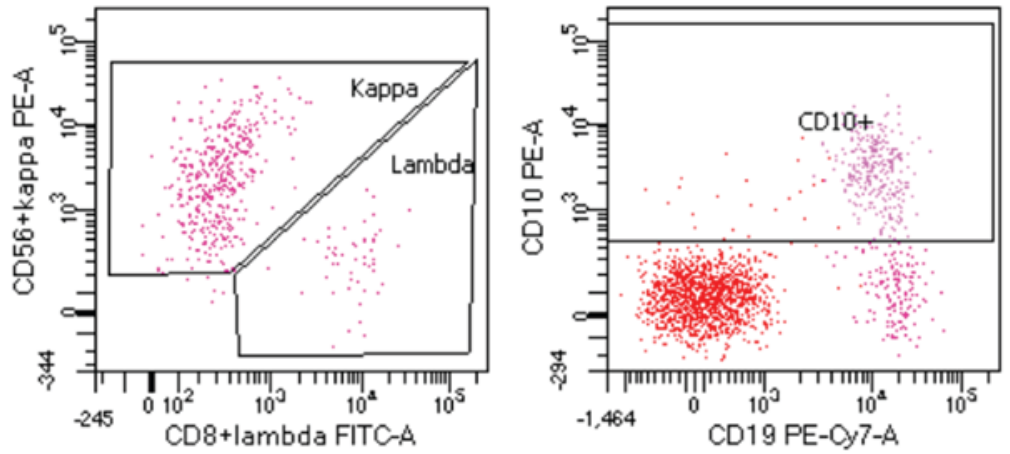

Figure 2. Flow cytometry of B-cells from a bone marrow biopsy at the time when gastric B-LBL was diagnosed demonstrating a kappa-chain restriction along with many CD19+ and CD10+ cells. These cells represent monoclonal immature B-cells characteristic for precursor B-LBL. B-LBL, B-lymphoblastic lymphoma.

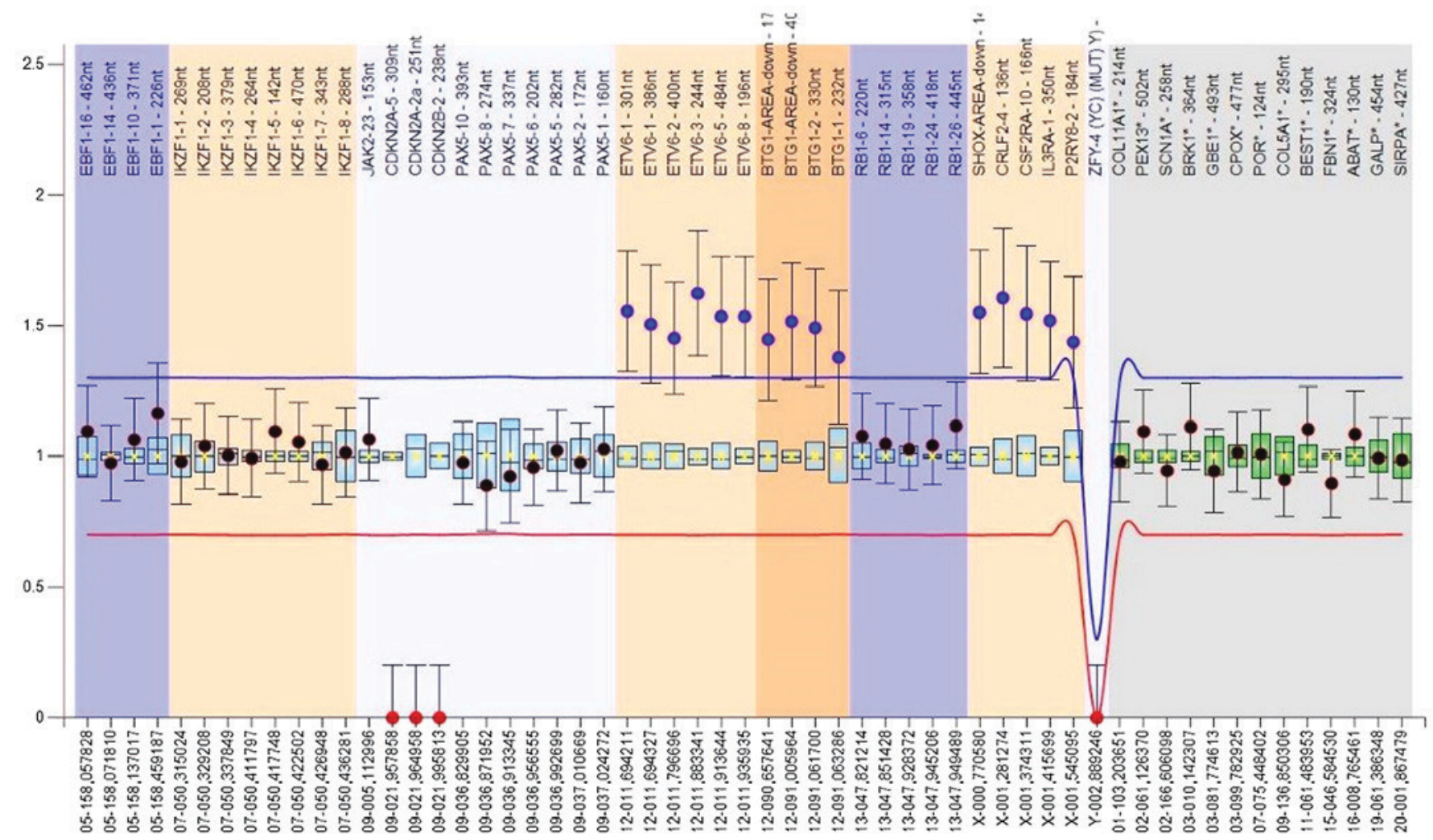

Figure 3. MLPA shows gain of chromosomes 12 and $\mathrm{X}$ and homozygous deletion of an area on 9p21, including CDKN2A/B.

A

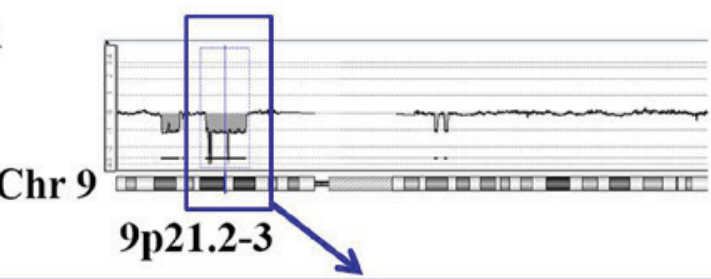

B

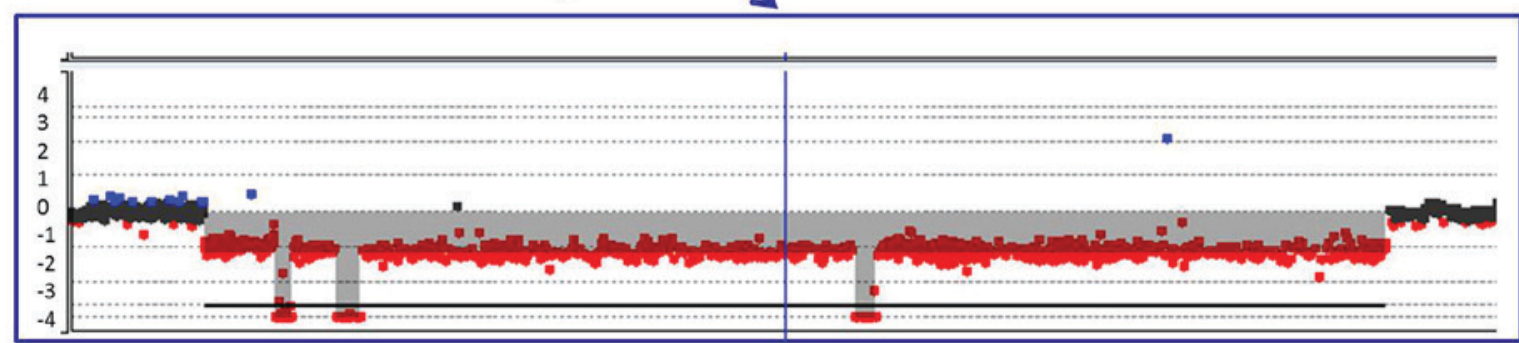

Figure 4. Array CGH analysis. (A) Whole chromosome view of chromosome 9 and (B) hemizygous and homozygous $C D K N 2 A / B$ deletions were observed. 
Accordingly, accurate diagnosis and prompt initiation of treatment adhering to high-risk protocols are therefore imperative. Future studies applying new technologies such as whole genome and RNA sequencing may provide new insight into the molecular pathways leading to B-LBL.

\section{Acknowledgements}

Not applicable.

\section{Funding}

No funding was received.

\section{Availability of data and materials}

All data generated or analyzed during the present study are included in this published article.

\section{Authors' contributions}

RE was involved in the study conception, writing of the manuscript, performed the literature review and contributed materials. LHM wrote the manuscript, and performed the histopathological analysis and literature review. MKA and SS contributed materials, and performed the genetics analysis and critical review. ECL contributed materials, and performed the histopathological analysis and critical review. LMRG contributed materials, and performed data interpretation and critical review. SH conceived and supervised the study, and performed the critical review.

\section{Ethics approval and consent to participate}

The patient provided written informed consent to participate. The study adheres to the declaration of Helsinki, and was approved by the National Committee on Health Research Ethics (H-B-2009-054).

\section{Patient consent for publication}

The patient provided written informed consent for publication.

\section{Competing interests}

The authors declare that they have no competing interests.

\section{References}

1. Bassan R, Maino E and Cortelazzo S: Lymphoblastic lymphoma: An updated review on biology, diagnosis, and treatment. Eur J Haematol 96: 447-460, 2016.

2. Thakker MM, Rubin PA and Chang E: Pre-B-cell acute lymphoblastic leukemia presenting as an orbital mass in an 8-month-old. Ophthalmology 113: 343-346, 2006.

3. Chou YC, Shih IH, Yang CP, Kuo TT and Hong HS: Concurrent mycosis fungoides and precursor B cell lymphoblastic lymphoma in a 6-year-old child. Pediatr Dermatol 22: 23-25, 2005.

4. Alford MA, Nerad JA, Conlan RM, Comito M and Giller RH: Precursor B-cell lymphoblastic lymphoma presenting as an orbital mass. Orbit 18: 17-24, 1999.

5. Faridpooya K, Mulder MM, Merks JH, de Smet MD, Pals ST and Saeed P: Precursor B lymphoblastic lymphoma of the orbit in a child: An unusual presentation of a non-Hodgkin lymphoma. Orbit 25: 153-157, 2006.
6. Ferry JA, Fung CY, Zukerberg L, Lucarelli MJ, Hasserjian RP, Preffer FI and Harris NL: Lymphoma of the ocular adnexa: A study of 353 cases. Am J Surg Pathol 31: 170-184, 2007.

7. Ramamoorthy J, Jain R, Trehan A, Saxena AK and Ahluwalia J: Orbital mass in a child with acute lymphoblastic leukemia: A case report and review of the literature. J Pediatr Hematol Oncol 38: 646-648, 2016.

8. Watkins LM, Carter KD and Nerad JA: Ocular adnexal lymphoma of the extraocular muscles: Case series from the University of Iowa and review of the literature. Ophthalmic Plast Reconstr Surg 27: 471-476, 2011.

9. Chin EK, Granier A, Hunter AA III, Dwyre DM and Lin LK: Extraocular muscle enlargement leading to the diagnoses of Burkitt lymphoma and acquired immune deficiency syndrome. Ophthalmic Plast Reconstr Surg 29: e157-e159, 2013.

10. Dias JS, Dittrich MA, Sousa JM, Teixeira LF and Manso PG: Medial rectus muscle myositis as an atypical presentation of mucosa-associated lymphatic tissue lymphoma: A case report. Arq Bras Oftalmol 77: 116-168, 2014.

11. Guo PD, Xian JF, Man FY, Liu ZH, Yan F, Zhao J and Wang ZC: Magnetic resonance imaging features of extraocular muscle lymphoma in five cases. Chin Med J (Engl) 129: 2384-2385, 2016.

12. Stenman L, Persson M, Enlund F, Clasen-Linde E, Stenman G and Heegaard S: Primary orbital precursor T-cell lymphoblastic lymphoma: Report of a unique case. Mol Clin Oncol 5: 593-595, 2016.

13. Jain P, Kantarjian H, Jain N, Short NJ, Yin CC, Kanagal-Shamanna R, Khoury J, Konopleva M, Sasaki K, Kadia TM, et al: Clinical characteristics and outcomes of previously untreated patients with adult onset T-acute lymphoblastic leukemia and T-lymphoblastic lymphoma with hyper-CVAD based regimens. Am J Hematol 92: E595-E597, 2017.

14. Toft N, Birgens H, Abrahamsson J, Bernell P, Griškevičius L, Hallböök H, Heyman M, Holm MS, Hulegårdh E, Klausen TW, et al: Risk group assignment differs for children and adults 1-45 yr with acute lymphoblastic leukemia treated by the NOPHO ALL-2008 protocol. Eur J Haematol 90: 404-412, 2013.

15. Mikkelsen LH, Andreasen S, Melchior LC, Persson M, Andersen JD, Pereira V, Toft PB, Morling N, Stenman G and Heegaard S: Genomic and immunohistochemical characterisation of a lacrimal gland oncocytoma and review of literature. Oncol Lett 14: 4176-4182, 2017.

16. Schejbel L, Schmidt IM, Kirchhoff M, Andersen CB, Marquart HV, Zipfel P and Garred P: Complement factor $\mathrm{H}$ deficiency and endocapillary glomerulonephritis due to paternal isodisomy and a novel factor $\mathrm{H}$ mutation. Genes Immun 12: 90-99, 2011.

17. Björklund E, Mazur J, Söderhäll S and Porwit-MacDonald A: Flow cytometric follow-up of minimal residual disease in bone marrow gives prognostic information in children with acute lymphoblastic leukemia. Leukemia 17: 138-148, 2003.

18. Bonn BR, Rohde M, Zimmermann M, Krieger D, Oschlies I, Niggli F, Wrobel G, Attarbaschi A, Escherich G, Klapper W, et al: Incidence and prognostic relevance of genetic variations in T-cell lymphoblastic lymphoma in childhood and adolescence. Blood 121: 3153-3160, 2013.

19. Swerdlow SH, Campo E, Harris NL, Jaffe ES, Pileri SA, Stein H and Thiele J (eds): WHO classification of tumours of haematopoietic and lymphoid tissues, Revised 4th edition. Lyon, IARC, 2017.

20. Safavi S, Hansson M, Karlsson K, Biloglav A, Johansson B and Paulsson K: Novel gene targets detected by genomic profiling in a consecutive series of 126 adults with acute lymphoblastic leukemia. Haematologica 100: 55-61, 2015.

21. Ou Z, Sherer M, Casey J, Bakos HA, Vitullo K, Hu J, Friehling E, Gollin SM, Surti U and Yatsenko SA: The genomic landscape of PAX5, IKZF1, and CDKN2A/B alterations in B-cell precursor acute lymphoblastic leukemia. Cytogenet Genome Res 150: 242-252, 2016.

This work is licensed under a Creative Commons Attribution-NonCommercial-NoDerivatives 4.0 International (CC BY-NC-ND 4.0) License. 